Mirai. Estudios Japoneses

ISSN-e: 2531-145X

\title{
El certificado de pareja del mismo sexo en Japón: análisis sociopolítico de los principales factores tras su aprobación regional e impedimentos para su establecimiento a nivel nacional.
}

\author{
Carla Labat de Hoz
}

Recibido: 28 de enero de 2019/ Aceptado: 15 de abril de 2019

Resumen. Establecidos por primera vez en 2015 en los distritos de Shibuya y Setagaya (Tokio), los certificados de pareja del mismo sexo son la primera muestra de reconocimiento legal de los derechos de la comunidad LGBT en Japón. En este artículo se realiza un análisis de los principales factores sociopolíticos que han posibilitado la aprobación de los certificados en diversas regiones del país, así como de los impedimentos existentes para su establecimiento a nivel nacional. La emisión local de los certificados se debe en gran medida al interés económico y político de ciertos sectores de la población japonesa y no a una demanda social real, pues apenas se ha producido el desarrollo de un activismo ideológico LGBT en el país. Por ello, su establecimiento a nivel nacional resulta poco probable en un futuro inmediato. No obstante, los certificados suponen un importante avance en el reconocimiento de los derechos de las minorías sexuales en Japón.

Palabras clave: LGBT; certificado de pareja del mismo sexo; minorías sexuales; arai; Japón.

[en] Same-Sex Partnership Certificate in Japan: A Sociopolitical Analysis of Key Factors Behind its Regional Approval and the Main Impediments to its Implementation at a National Level.

\begin{abstract}
Same-sex partnership certificates, established for the first time in 2015 in the districts of Shibuya and Setagaya (Tokyo), are the first legal recognition of the rights of the LGBT community in Japan. This article carries out an analysis of the main sociopolitical factors behind the establishment of the certificates in several regions of the country as well as of the existing impediments to their establishment on a national level. The local granting of the certificates is largely due to economic and political interests of certain sectors of the Japanese population. Therefore, it has not been prompted by a social demand as the development of an ideologically motivated LGBT activism in the country has hardly developed. The establishment of the certificates at a national level is thus an unlikely outcome in the near future. Nevertheless, these certificates represent an important step in the recognition of the rights of sexual minorities in Japan.
\end{abstract}

Keywords: LGBT; same-sex partnership certificate; sexual minorities; arai; Japan.

Sumario. 1. Introducción. 2. Trabas legales y estrategias de las parejas homosexuales en el contexto japonés. 3. Factores tras la aprobación de los certificados de pareja de mismo sexo. 4. Impedimentos para la nacionalización y difusión del certificado de pareja del mismo sexo en Japón. 5. Conclusión. Bibliografía

\footnotetext{
Universidad de Heidelberg - Universidad de Kioto.

carlalabat@hotmail.com
} 
Cómo citar: Labat de Hoz, C. (2019).El certificado de pareja del mismo sexo en Japón: análisis sociopolítico de los principales factores tras su aprobación regional e impedimentos para su establecimiento a nivel nacional, en Mirai. Estudios Japoneses 3(2019), 219-234.

\section{Introducción}

El artículo 21 de la Carta de los Derechos Fundamentales de la Unión Europea (18 de diciembre de 2000) proscribe la discriminación de toda persona por motivos de orientación sexual ${ }^{2}$. En el marco legal internacional, la discriminación por motivos de orientación sexual y/o identidad de género también queda prohibida por el derecho internacional de los derechos humanos ${ }^{3}$. Sin embargo, los derechos de las minorías sexuales ocupan un espacio jurídico más incierto al quedar recogidos implícita o explícitamente en diferentes tratados y acuerdos internacionales.

Los Principios de Yogyakarta sobre la Aplicación de la Legislación Internacional de Derechos Humanos en relación con la Orientación Sexual y la Identidad de Género (2007) han tenido un valor crucial por su carácter pionero. Pese a no ser vinculantes, definieron el marco de protección en cuestiones de orientación sexual y de género establecido en términos generales por los derechos humanos ${ }^{4}$. Con todo, en el reconocimiento de los derechos de las minorías sexuales, las Resoluciones 17/19 y 27/32 del Consejo de Derechos Humanos de Naciones Unidas han supuesto el mayor avance hasta la fecha.

Dichas resoluciones, tituladas "Derechos Humanos, Orientación Sexual e Identidad de Género de la ONU", fueron aprobadas en 2011 y 2014 respectivamente y contaron con el apoyo de Japón 5 . No obstante, el mismo año en que la Resolución 27/32 fue adoptada, el país asiático recibió indicaciones por parte del organismo internacional de desarrollar medidas integradoras del colectivo $\mathrm{LGBT}^{6}$ ante el escaso avance a nivel nacional que se había producido desde $2011^{7}$. En concreto, la ONU dio especial relevancia a la ausencia de un sistema de reconocimiento legal de las parejas del mismo sexo. A diferencia de otros Estados miembros con un nivel de desarrollo socioeconómico similar, en Japón estas parejas aún no pueden formalizar su relación a través del matrimonio $\mathrm{u}$ otras instituciones legales a nivel estatal.

Fue por tanto una gran sorpresa para muchos cuando el 31 de marzo de 2015 el distrito de Shibuya (Tokio) anunció su plan de emitir certificados en reconocimiento de los derechos legales de las parejas homosexuales. A esta moción se sumó el vecino distrito de Setagaya y ambos comenzaron a emitir los "certificados de pareja

\footnotetext{
Véase Carta de los Derechos Fundamentales de la Unión Europea [EUR-Lex (2016)].

ACNUR (2014): 11-12.

4 Véase Principios de Yogyakarta sobre la Aplicación de la Legislación Internacional de Derechos Humanos en Relación con la Orientación Sexual y la Identidad de Género [International Commission of Jurists (2007)].

Chi, N. (2016): 76.

6 Las siglas LGBT hacen referencia tanto a las personas lesbianas, gais, bisexuales y trangénero como a la comunidad que conforman. En este artículo el término será empleado teniendo en consideración también otras formas de orientación sexual y de género, como la pansexualidad o la asexualidad. Actualmente se está difundiendo el uso de diversas ampliaciones de las siglas LGBT en las que diversas minorías sexuales quedan recogidas; no obstante, como no se ha fijado el uso de ninguna de ellas por el momento, se ha preferido utilizar LGBT teniendo en consideración esta extensión de su significado.

Matsunobu, H. (2016): 71.
} 
del mismo sexo" (dōsei pātonāshippu shōmeisho) ${ }^{8}$ el 5 de noviembre del mismo año, convirtiéndose de forma conjunta en las primeras regiones del país en hacerlo9. La ciudad de Iga, en la prefectura de Mie, se convirtió en el tercer gobierno local en legalizar los certificados el 1 de abril de 2016. Seguidamente, la ciudad de Takarazuka, en la prefectura de Hyōgo, comenzó a emitir los certificados el 1 de junio y la ciudad de Naha, en la prefectura de Okinawa, se convirtió el 8 de julio en la primera capital de prefectura en aceptarlos. Los últimos avances en el establecimiento de estos certificados se han dado el 1 de junio de 2017 en Sapporo (capital de la prefectura de Hokkaidō) ${ }^{10}$ y el 1 de abril de 2018 en Fukuoka (capital de la prefectura de Fukuoka $)^{11}$. Especialmente notorio ha sido el caso de la primera ciudad, puesto que se trata de una de las veinte mayores ciudades del país.

A pesar de que la legalización del matrimonio homosexual es todavía una realidad poco probable en el futuro inmediato del país ${ }^{12}$, esta difusión regional de los certificados de pareja del mismo sexo parece indicar que su establecimiento a nivel nacional podría darse en los próximos años. En este artículo se presenta un análisis tanto de los diferentes factores sociopolíticos que han permitido la creación de los mencionados certificados como de los principales impedimentos a los que se han enfrentado sus promotores a fin de determinar la viabilidad de dicho desarrollo. El fenómeno de la aprobación de los certificados del mismo sexo es atendido, por lo tanto, desde una perspectiva antropológica y sociológica en vez de una jurídica o regulatoria. Así mismo, en este estudio se ha tratado de determinar a través de fuentes académicas y periodísticas el acogimiento de la medida tanto por la comunidad LGBT como por el público general.

A fin de atender todas estas cuestiones, el artículo se divide en tres partes. En una primera sección se presentan los sistemas alternativos de reconocimiento legal desarrollados por la comunidad LGBT japonesa y sus principales limitaciones a fin de establecer el contexto en el cual se está produciendo el establecimiento de los certificados de pareja. A continuación, se lleva a cabo un estudio de los principales factores sociopolíticos y económicos tras su aprobación en algunas regiones del país. En una tercera sección se analizan los principales impedimentos para la difusión y nacionalización de los certificados de pareja del mismo sexo en Japón. Finalmente, se presentan brevemente las conclusiones derivadas de lo puntos tratados con anterioridad a forma de conclusión.

\section{Trabas legales y estrategias de las parejas homosexuales en el contexto japonés}

A finales de la década de 1990, en algunos países europeos, Canadá y ciertas regiones de Estados Unidos los derechos legales de las parejas homosexuales comenzaron

\footnotetext{
8 Aunque bajo este término quedan recogidos todos los modelos de certificado establecidos en los distritos de Shibuya y Setagaya y en las ciudades de Iga, Takarazuka, Naha, Sapporo y Fukuoka, a nivel administrativo se usan diferentes términos en cada región. En el caso de Setagaya, Iga, Takarazuka, Sapporo y Fukuoka se denomina "recibo" (juryōshō) mientras que en Naha se considera un "certificado de registro" (tōroku shōmei-sho). En el distrito de Shibuya se utiliza exclusivamente el término general [Itō, H. (2018)].

9 Chi, N. (2016): 75-81; Matsunobu, H. (2016): 73.

10 Kazuhiro, S. (2016).

11 Itō, H. (2018).

12 Arai, Y. (2014): 154; Lunsing, W. (2005): 147; Sørensen, J. D. (2016): 28; Suganuma, K. (2006).
} 
a ser reconocidos por medio de la emisión de certificados de pareja. Así mismo, diversos países establecieron las uniones de hecho y en 2001 Países Bajos se convirtió en el primer país del mundo donde el matrimonio homosexual se hizo legal ${ }^{13}$.

Estos desarrollos impulsaron a comienzos del nuevo siglo la aparición de un debate entre académicos y juristas japoneses sobre la conveniencia de una posible legalización de dichos enlaces en el país o el establecimiento de certificados de pareja del mismo sexo ${ }^{14}$. La comunidad LGBT había comenzado a reconocer la existencia de una serie de "barreras legales" (seido no kabe) por las que las parejas del mismo sexo quedan excluidas en el país tanto de los derechos como de los beneficios de los que gozan las parejas heterosexuales al no poder formalizar su relación a través de la institución matrimonial ${ }^{15}$. Por ejemplo, uno de los problemas más acuciantes que diversos autores y miembros de las minorías sexuales han denunciado es la imposibilidad de acceso en pareja a una vivienda, ya sea pública o privada.

El acceso individual a una vivienda pública (kōei jūtaku) es posible en Japón para cualquier mujer mayor de 50 años, hombre mayor de 60 años o persona discapacitada que cumpla con los requerimientos de la ley en cuanto a renta mínima. Aunque bajo esta premisa las minorías sexuales pueden beneficiarse de este sistema estatal, no pueden hacerlo con sus parejas. La Ley de Vivienda Pública establece como una condición necesaria en su artículo 27 párrafo 5 que los residentes de las viviendas públicas sean parientes que vivieron juntos de forma previa al traslado. Por ende, reconoce a las personas que mantienen una relación a través de lazos biológicos o a través de instituciones legales como el matrimonio y la adopción. La ley también reconoce a aquellas parejas que planean casarse o que mantienen una relación similar a la matrimonial. Puesto que las parejas del mismo sexo no pueden formalizar su relación de dicha manera, el corpus legal excluye implícitamente a las parejas homosexuales ${ }^{16}$.

En lo concerniente a la búsqueda de vivienda privada, es necesario tener en consideración que se trata de una de las principales dificultades a la que deben de hacer frente los miembros de la comunidad LGBT en Japón, especialmente las parejas. E1 vendedor o arrendatario de un inmueble tiene derecho a preguntar por la naturaleza de la relación que mantienen sus compradores o inquilinos y desestimar su oferta por ello. Además, puesto que su relación no es reconocida de forma oficial, las parejas del mismo sexo no pueden solicitar préstamos hipotecarios de forma conjunta ${ }^{17}$, lo que dificulta en muchas ocasiones el acceso al capital necesario para la adquisición de un inmueble.

Otro problema acusado por las parejas homosexuales es la falta de reconocimiento por parte de las empresas aseguradoras. Los miembros de la comunidad LGBT no pueden beneficiarse de las pensiones o seguros de vida contratados por sus parejas a empresas nacionales o extranjeras puesto que su relación no ha sido formalizada legalmente ${ }^{18}$. Esta condición no queda recogida en ningún corpus legislativo oficial, sino que es un requisito establecido en las bases de las propias compañías asegu-

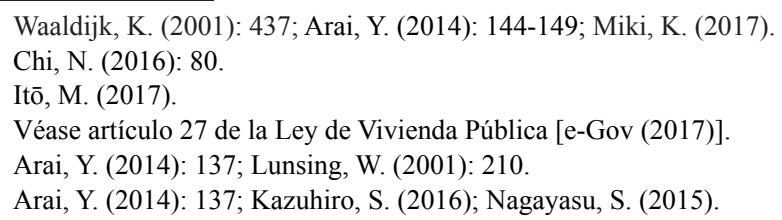


radoras ${ }^{19}$. Así mismo, al no estar reconocida oficialmente su relación, las parejas homosexuales no tienen derechos hereditarios en caso de defunción de la otra parte ni pueden acceder a deducciones conyugales en el pago de impuestos. Tampoco pueden beneficiarse de los descuentos familiares que otorgan algunas compañías a sus clientes ni pueden obtener las ventajas laborales que algunas empresas destinan a sus trabajadores casados ${ }^{20}$.

Finalmente, es necesario mencionar que, en caso de defunción o de emergencia médica de uno de los miembros de la pareja, la otra parte no puede ejercer como su representante legal. Por tanto, no pueden tomar decisiones concernientes a tratamientos médicos, medidas para la prolongación de la vida o la donación de órganos cuando su pareja no se encuentra capacitada física o mentalmente para hacerlo. También carecen del derecho de visita en caso de hospitalización, puesto que no son reconocidos como miembros familiares ${ }^{21}$.

Todas estas cuestiones han planteado serias dificultades a las minorías sexuales, quienes han tratado de recurrir a vacíos legales o a sistemas alternativos al matrimonio para poder formalizar sus relaciones de cara a las instituciones y empresas estatales. Dentro de estas medidas, dos prácticas que han destacado tradicionalmente han sido la elaboración de actas notariales y la adopción entre adultos.

La primera cuestión consiste en el establecimiento de una relación contractual a través de un notario. En el acta, cada miembro de la pareja reconoce al otro como su representante legal en caso de incapacitación física o mental en temas como la administración de la propiedad o los cuidados médicos. Estos "acuerdos de convivencia conjunta" (kyoudou seikatsu sengen) fueron desarrollados de forma previa al reconocimiento de las uniones de hecho en Japón por aquellas parejas heterosexuales que no querían recurrir a la institución matrimonial para proteger su relación legalmente $^{22}$. No obstante, la Corte Suprema japonesa reconoce actualmente los mismos derechos conyugales tanto para las parejas casadas como para las parejas de hecho heterosexuales. Por tanto, estas actas notariales son más comunes entre parejas homosexuales hoy día ${ }^{23}$.

El sistema de adopción, recogido en el Libro IV Título III del Código Civil ${ }^{24}$, se conoce como yōshi engumi y comprende tanto una forma de adopción "plena" (tokubetsu) como una "simple" (futsū). Esta segunda forma de adopción, a la que se acogen las parejas homosexuales, es posible cuando al menos uno de los integrantes ha alcanzado la mayoría de edad y quien adopta es mayor que el adopta$\mathrm{do}^{25}$. Bajo este sistema, la pareja puede convertirse en una familia, con todos los derechos legales que a estas se les reconoce. Por ejemplo, tras la adopción, los integrantes pueden usar el mismo nombre de familia, tienen responsabilidad de asistencia mutua y poder de representación legal y gozan de derechos hereditarios. Aunque diversos estudios indican que las lesbianas recurren con mayor frecuencia a las actas notariales para formalizar sus relaciones, la comunidad LGBT

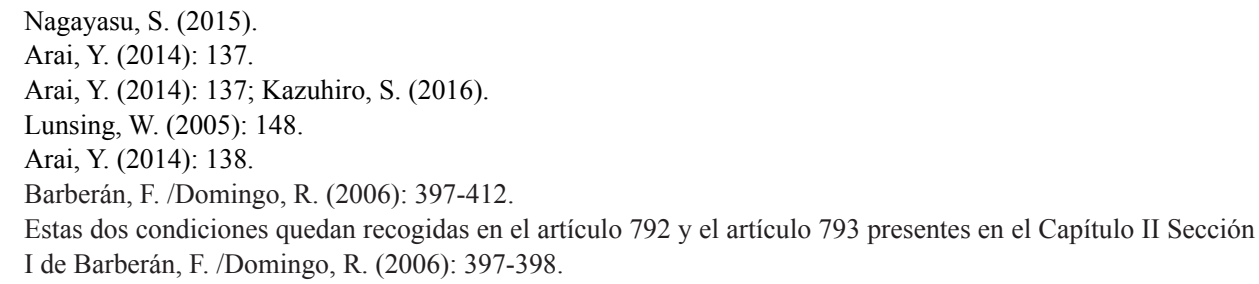


parece preferir este segundo sistema en términos generales por todas sus ventajas legales ${ }^{26}$.

No obstante, la adopción también plantea una serie de problemas a las parejas homosexuales. Si bien algunos autores defienden que esta manera de formalizar su unión es tan fuerte como la matrimonial ${ }^{27}$, lo cierto es que cuenta con importantes limitaciones legales y no libera completamente a la pareja de su situación de inestabilidad. Las parejas que recurren a la adopción simple no pueden adoptar menores posteriormente como pareja puesto que su relación queda definida como la de un padre y un hijo o una madre y una hija. Así mismo, en caso de que se llegase a legalizar el matrimonio homosexual, estas personas ya no podrían acceder a él ${ }^{28}$. Además, debe tenerse en cuenta que la adopción podría ser anulada si es considera "dañina para la moral pública". Este caso podría darse, por ejemplo, si un familiar de los cónyuges denuncia que la intención real no era la adopción y que los integrantes mantienen una relación de pareja ${ }^{29}$.

Las actas notariales y la adopción han sido y son medidas comúnmente usadas por las minorías sexuales para regularizar sus relaciones; no obstante, esto no impide que algunos de sus integrantes las rechacen al considerar que no permiten a la pareja constituirse como tal de forma real. Así mismo, muchos han denunciado el daño emocional que supone el no poder obtener de forma honesta un reconocimiento oficial de su relación y el tener que recurrir a vacíos legales o al engaño ${ }^{30}$. Sin embargo, las parejas del mismo sexo en Japón apenas se han movilizado para defender su derecho al acceso a la institución matrimonial u otras formas de reconocimiento legal. Una cuestión que en parte responde al escaso desarrollo de un movimiento LGBT en el país.

La ausencia de una persecución legal o religiosa de las minorías sexuales ha sido uno de los motivos por los cuales el activismo LGBT apenas ha tomado forma en Japón. Al no haber sido atacada activamente por las fuerzas de autoridad del país, la comunidad LGBT no se ha visto impelida a emprender medidas en la defensa de sus derechos, a pesar de que estos son considerablemente escasos. Con la crisis del VIH en la década de los noventa, se dio cierta movilización por parte de las minorías sexuales mayoritariamente vinculada a la lucha contra los estereotipos negativos del colectivo presentados por los medios de comunicación. No obstante, a diferencia de lo ocurrido en otros países como Estados Unidos, la crisis no inició debates sobre el matrimonio homosexual $\mathrm{u}$ otras formas de reconocimiento legal de las parejas homosexuales ${ }^{31}$.

Con el cambio de siglo, esta actitud pasiva por parte de la comunidad LGBT se ha visto propiciada por el desarrollo de tendencias individualistas en la sociedad japonesa y la reducción del heteronormativismo ${ }^{32}$, causada por la crisis del modelo

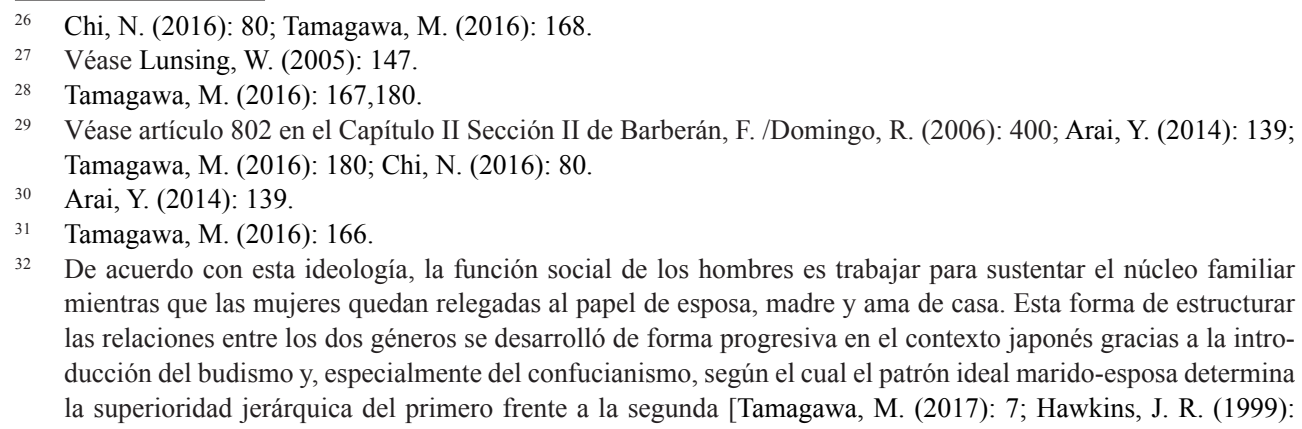
mientras que las mujeres quedan relegadas al papel de esposa, madre y ama de casa. Esta forma de estructurar las relaciones entre los dos géneros se desarrolló de forma progresiva en el contexto japonés gracias a la introducción del budismo y, especialmente del confucianismo, según el cual el patrón ideal marido-esposa determina la superioridad jerárquica del primero frente a la segunda [Tamagawa, M. (2017): 7; Hawkins, J. R. (1999): 
del salaryman establecido en la posguerra ${ }^{33}$. No obstante, el verdadero motivo por el cual el colectivo no ha promovido de forma más activa la legalización del matrimonio homosexual u otros modos de reconocimiento legal parece ser la baja aceptación social que estas medidas recibirían ${ }^{34}$. En otras palabras, las minorías sexuales no promueven la regulación oficial de sus uniones puesto que temen el consecuente estigma social que esto podría suponer, debido a la pervivencia de un modo de discriminación otonashi y uchi hacia la comunidad LGBT en Japón.

La discriminación otonashi ("sin hacer ruido") consiste en el silenciamiento de las minorías sexuales a través de la creación de un espacio público inherentemente heteronormativo. Los miembros de la comunidad LGBT no pueden expresar abiertamente su orientación sexual o de género en este contexto puesto que, además, la expresión sexual es considerada un ámbito del individuo perteneciente a lo privado. Por lo tanto, estas personas tienen que hacer frente a una sociedad que las considera heterosexuales de facto. La discriminación uchi ("familiar") se basa en el rechazo que las minorías sexuales padecen por parte de su núcleo familiar. El ámbito privado permite a los integrantes de la familia expresar abiertamente sus sentimientos homofóbicos acallados en el ámbito público ${ }^{35}$.

Puesto que tanto la discriminación otonashi como la discriminación $u c h i$ no se materializan de forma explícita, no se puede demostrar su existencia con facilidad. Los miembros de la comunidad LGBT tampoco han respondido activamente ante estas formas de discriminación en defensa de sus derechos. Solo recientemente parece que se están produciendo avances en el reconocimiento legal de las parejas homosexuales con la aprobación de los certificados de pareja del mismo sexo en algunas regiones de Japón. Es necesario por tanto considerar cuales han sido los factores que han posibilitado el establecimiento de dichos certificados a fin de determinar posteriormente su posible aplicación a nivel nacional.

\section{Factores tras la aprobación de los certificados de pareja de mismo sexo}

Las primeras demandas en favor del establecimiento de un certificado para parejas del mismo sexo se produjeron durante la primera década del siglo veintiuno y sus protagonistas fueron tanto académicos como activistas y juristas pertenecientes al colectivo LGBT. A través de obras como Domesutikku pātonā: atarashî kankei no sōzō ni mukete (Pareja de hecho: hacia la creación de una nueva relación) ${ }^{36}$, publicada en 2004, trataron de hacer llegar a un público más amplio su defensa de un sistema de pareja igualitario para homosexuales y heterosexuales contrario al establecido por el sistema de registro familiar. No obstante, el debate concerniente a una posible aprobación a nivel nacional del certificado no ha tomado forma hasta la aprobación de la medida en los distritos de Shibuya y Setagaya en Tokio ${ }^{37}$.

94-162; Tamagawa, M. (2016):172].

33 Arai, Y. (2014): 122-126; McLelland, M./ Suganuma, K. (2009): 340; Suganuma, K. (2006).

34 Arai, Y. (2014): 143; Tamagawa, M. (2016): 173.

35 McLelland, M. (2005): 103-104; Tamagawa, M. (2017): 8; McLelland, M./ Suganuma, K. (2009): 329; Arai, Y. (2014): 130, 149.

36 Traducción realizada por la autora de este artículo.

37 Tamagawa, M. (2016): 174; Chi, N. (2016). 
A diferencia de en otros países donde las quejas de la comunidad LGBT y el activismo del colectivo han sido factores fundamentales para el desarrollo de las reformas legales en su defensa, en Japón la emisión de los certificados de pareja del mismo sexo no puede considerarse su consecuencia. Es cierto que, hasta cierto punto, las minorías sexuales han intervenido tanto en el proceso de decisión como en la elaboración de los certificados. Sin embargo, su desarrollo ha sido impulsado mayoritariamente por miembros de asambleas regionales y otras personalidades del gobierno con conocimiento de los derechos de las minorías sexuales en otros países ${ }^{38}$ y que forman parte de los llamados arai o "aliados" 39 .

En el contexto japonés, es poco común que los ciudadanos provoquen cambios en la administración ${ }^{40}$. Por ello, la difusión de los certificados de pareja del mismo sexo a otras partes de Japón se está produciendo con mayor lentitud que en otros países donde medidas similares se han extendido del ámbito regional al nacional. Los certificados han surgido como una iniciativa de ciertos gobiernos locales sin tener un apoyo social real. De las siete regiones donde se ha aprobado su emisión, solo en Sapporo parece que haya sido consecuencia directa de la acción conjunta de abogados defensores de los derechos humanos y grupos activistas LGBT ${ }^{41}$.

Por tanto, a excepción de Sapporo, el establecimiento de los certificados de pareja del mismo sexo se ha debido en gran medida al esfuerzo individual de ciertas figuras dentro de algunas asambleas regionales. Estas personalidades se han aprovechado de la reticencia del Partido Liberal Democrático (LDP) ${ }^{42}$ a atender cualquier asunto concerniente a las minorías sexuales para llevar a cabo cambios en las políticas locales. Al presentar dichos cambios como una respuesta necesaria a las necesidades específicas de los miembros de sus administraciones en vez de como demandas de derechos civiles con base ética o moral, han podido llevarlos a cabo sin tener que hacer frente a una fuerte oposición por parte del gobierno central ${ }^{43}$.

Por ejemplo, en Shibuya, la primera región en establecer los certificados, parece haber sido fundamental la figura de Ken Hasebe, gerente del distrito desde abril de 2015 hasta la fecha. El político, que no está afiliado a ningún partido, se convirtió en un arai tras entrar en contacto con diversos miembros notorios y activistas de las minorías sexuales. Fue entonces cuando decidió impulsar la creación de los certificados en el distrito $^{44}$. En vez de plantear la cuestión como un asunto de derechos humanos, lo hizo

38 Matsunobu, H. (2016): 74.

39 Los arai "[v]an un paso más allá de ser amables, son personas que apoyan más activamente, personas que buscan la mejora del estatus social y de los derechos de las personas LGBT, significa personas que cooperan en el movimiento para lograr la igualdad" [Miki, K. (2017): 30].

40 Kazuhiro, S. (2016); Hitofude, H. (2017).

${ }_{41}$ Tras la aprobación del certificado de pareja del mismo sexo en Shibuya y Setagaya, se formó en Sapporo el grupo activista Domestic Partnership in Sapporo, cuyo principal objetivo ha sido el establecimiento de dicho certificado en la ciudad. El grupo de apoyo recogió firmas y presentó su demanda al ayuntamiento, consiguiendo la creación de un certificado en junio de 2017 [Kazuhiro, S. (2016); Hitofude, H. (2017)].

42 El LDP es la principal fuerza política de Japón y desde su creación en 1955 ha gobernado el país de forma ininterrumpida a excepción del periodo entre 1993 y 1994 y el periodo entre 2009 y 2012. Pese a que se trata de una formación política que por su amplitud recoge ideologías muy diferentes, en términos generales se trata de un partido político muy conservador en su concepción de la familia y el género [Arai, Y. (2014): 138; LDP (2018)].

43 Yasuo, T. (2017): 18.

44 En 2012, cuando aún era solo un miembro de la asamblea del distrito, propuso la elaboración de los certificados a Toshitake Kuwabara, el por aquel entonces gerente, quien decidió en 2014 crear la Comisión de Investigación sobre el Establecimiento de la Ordenanza de Shibuya por la Promoción del Respeto a la Diversidad Comunitaria. La comisión contó con ocho miembros, entre ellos abogados, académicos y miembros independientes de 
como si se tratase de una cuestión de imagen e "internacionalización" de la ciudad. Los certificados desarrollarían la comunidad como centro cultural a la par que Londres, Nueva York u otros grandes centros urbanos. Aunque la intención real de la medida era promover el reconocimiento social de las parejas homosexuales, Ken indicó que los certificados respondían antes que nada a las necesidades reales de los residentes del distrito. Es decir, sus argumentos fueron fundamentalmente pragmáticos y evitó que los documentos requiriesen cambiar el sistema de registro familiar ${ }^{45}$.

De la misma manera, en el distrito de Setagaya ha sido crucial la figura del gerente del distrito, Nobuto Hosaka, quien accedió al cargo en 2011 y fue reelegido en 2015. Es considerado un importante arai no solo por sus declaraciones a favor de aumentar la visibilización del colectivo LGBT y su participación en numerosos eventos pride $^{46}$, sino también por haber posibilitado la emisión de los certificados. Puesto que la asamblea del distrito estaba dominada por miembros del conservador LDP, decidió llevar a cabo su establecimiento como una directriz administrativa (shi$d \bar{o} y \bar{o} k o)^{47}$.

No obstante, debe entenderse que el establecimiento de los certificados no se trata de un fenómeno aislado en cada región motivado exclusivamente por el esfuerzo individual de ciertos particulares. Un factor que parece estar facilitando la difusión de la medida a nuevos territorios de Japón es la interacción entre los miembros de estas asambleas regionales. Por ejemplo, la activista y política Kamikawa Aya ${ }^{48}$ apoyó firmemente a Ken Hasebe cuando anunció oficialmente la emisión de los certificados en Shibuya y promovió que estos fueran establecidos en el distrito de Setagaya. Así mismo, sus interacciones con el gerente alcalde de Takarazuka, Tomoko Nakagawa, fueron fundamentales para que el 1 de junio de 2016 se aprobara la emisión de los certificados en dicha ciudad siguiendo el modelo de Setagaya. Bajo este sistema de shidō yōko también se sumaron a la medida las ciudades de Iga, Naha y, finalmente, Sapporo $^{49}$.

Junto a lo anteriormente expuesto, debe tenerse en consideración además que las iniciativas particulares de las administraciones locales se han visto impulsadas por motivos políticos y económicos. Aunque el gobierno central está dirigido actualmente por el LDP, algunos partidos minoritarios como el Partido Democrático de Japón (JDP), el Partido Kōmeitō, el Partido Socialdemócrata (PSJ) y el Partido Comunista de Japón (PCJ) han modificado recientemente sus manifiestos y proponen el reconocimiento de los derechos legales de las minorías sexuales pese a no contar con agendas concretas. En una encuesta sobre la legalización de las uniones homo-

la asamblea del distrito como Mari Okada, quien también había solicitado al gerente el establecimiento de los certificados [Yasuo, T. (2017): 19-21; Tsuji, K. (2016); Naohiko, H. (2016); Shusuke, M. (2015)].

45 Yasuo, T. (2017): 19-24.

46 Shusuke, M. (2015).

47 Las guías (shidō yōko) son decididas e implementadas de forma unilateral por el gerente del distrito. En Shibuya, el establecimiento de los certificados quedó recogido en una ordenanza (jōrei), que son emitidas tras ser sometidas a votación y aprobadas por mayoría en las asambleas de los distritos [Ayuntamiento del distrito de Setagaya (2015); Ayuntamiento del distrito de Shibuya (2015)].

48 Kamikawa Aya se convirtió en 2003 en la primera política abiertamente transgénero con un cargo en una oficina pública al convertirse en miembro de la asamblea municipal del distrito de Setagaya. En 2007, 2011 y 2015 fue reelegida para el cargo y es una figura clave en la defensa de los derechos de reconocimiento legal de las minorías sexuales [Chi, N. (2016): 83; Itakura, K. (2015): 26; McLelland, M./ Suganuma, K. (2009): 338; Keiji, H. (2017)].

49 Yasuo, T. (2017): 24-25. 
sexuales realizada a los diferentes partidos, solo el LDP se opuso de forma activa. Además, algunos de los integrantes de estos grupos políticos minoritarios son figuras fundamentales del activismo LGBT en el país ${ }^{50}$.

Así mismo, de acuerdo con algunos autores, en ciertos gobiernos locales se está difundiendo una política de "homonacionalismo" por la que se busca presentar a Japón como un país gay friendly. Esta política se ha visto especialmente fortalecida en vistas a los próximos Juegos Olímpicos de 2020, puesto que los patrocinadores oficiales de la competición requieren al país de acogida medidas para la inclusión de la comunidad LGBT. Considerando las sanciones realizadas a Rusia durante los Juegos Olímpicos de Invierno de 2014, se están promoviendo por ello políticas de integración desde la administración central. Esta consideración se ha extendido además al sector privado, especialmente a aquellas compañías nacionales que van a financiar la celebración del evento. Ejemplo de ello es Panasonic, que desde 2016 otorga a las parejas del mismo sexo poseedoras del certificado las mismas ventajas laborales que al resto de sus trabajadores casados. La empresa ha reconocido que el evento deportivo ha sido uno de los motivos para llevar a cabo este cambio en su política laboral interna ${ }^{51}$.

Por otro lado, se debe tener en cuenta además el factor económico como uno de los motores principales en la promoción del establecimiento de los certificados. El interés de las diferentes empresas nacionales e internacionales por atender a la demanda del colectivo LGBT en el país está propiciando reformas legales que facilitan su inclusión en las políticas de dichas entidades. En los resultados de su encuesta de 2015, Dentsu no solo hizo público que el 7,6\% de la población japonesa pertenece a una minoría sexual, sino que además estimó que dicho porcentaje supone un mercado de 5,94 trillones de yenes (más de 47.000 millones de euros) ${ }^{52}$.

En una sociedad donde el envejecimiento de la población y la baja natalidad suponen una reducción constante del mercado, atender a este sector de la población se ha vuelto crucial para algunas empresas, como las aseguradoras y las compañías matrimoniales, que han comenzado a conceder sus servicios a las parejas homosexuales ${ }^{53}$. Otras empresas también están comenzando a incluirlas en sus promociones y planes especiales, hasta el momento reservados a los matrimonios heterosexuales ${ }^{54}$. Con este tipo de medidas, las compañías no solo pretenden atender al colectivo LGBT, sino también atraer a aquellas personas que apoyan a dichas minorías por medio de una publicidad gay friendly. Como declaró Ayaka Asami, una de las principales investigadoras de Dentsu, "[e]l tiempo de focalizarse solo en los LGBT por su poder adquisitivo ya ha finalizado en Japón. El poder adquisitivo de los heterosexuales que apoyan a la comunidad LGBT está ampliando el mercado" 55 .

Establecer medidas para la integración de las minorías sexuales tiene además un importante poder de marketing para las compañías, pero solo ha sido posible tras

50 Por ejemplo, el Partido Democrático ha creado un comité para las minorías sexuales y tiene como afiliada a Kanako Otsuji, miembro de la Asamblea de la prefectura de Osaka y de la Cámara Alta del Parlamento. El Partido Socialdemócrata cuenta entre sus filas con Taiga Ishikawa, miembro de la Asamblea Local en el distrito de Toshima [Arai, Y. (2014): 138; Chi, N. (2016): 82-83].

51 Itakura, K. (2015): 18; Shirakawa, T. (2016), Masaru, S. (2016).

52 Dentsu (2015).

53 Nagayasu, S. (2015); Nikkei (2016).

${ }_{54}$ Shirakawa, T. (2016).

55 Mariko, T. (2015). 
la aprobación de los certificados ${ }^{56}$. Pese a que las empresas ya eran conscientes del valor del mercado LGBT, no tomaron ninguna iniciativa con anterioridad al no poder asegurar que estas medidas fuesen aceptadas por el grueso de la población. La aprobación de los certificados de pareja del mismo sexo era la señal que necesitaban de forma definitiva para comenzar a atender a este mercado.

\section{Impedimentos para la nacionalización y difusión del certificado de pareja del mismo sexo en Japón}

A pesar de la existencia de todos estos factores tras la aprobación de los certificados de pareja del mismo sexo en los distritos de Shibuya y Setagaya, así como en las ciudades de Iga, Takarazuka, Naha, Sapporo y Fukuoka, un debate a nivel nacional sobre la cuestión parece poco probable en un futuro inmediato. Especialmente si se tiene en consideración la postura que mantiene al respecto el gobierno central actual $^{57}$.

Aunque el vacío legal en lo que respecta a la comunidad LGBT ha posibilitado el establecimiento de los certificados por parte de los gobiernos locales, el LDP no apoya que medidas similares sean tomadas en el plano estatal al considerarlas subversivas de los valores conservadores tradicionales ${ }^{58}$. Puesto que además el grueso de la población no ha mostrado gran interés en promover los derechos de las minorías sexuales, el tema no es atendido en los debates electorales y, por tanto, los partidos políticos no se ven compelidos a pronunciarse sobre él. Esto favorece al LDP, pues puede mantener su postura sin tener que hacer frente a la oposición de otros partidos $^{59}$. Así mismo, la falta de homogeneidad entre los sistemas de certificado ya establecidos evidencia la enorme dificultad que supondría imponer la medida de forma común en todas las regiones.

No obstante, estas cuestiones no explican la lenta difusión de los certificados en las administraciones locales de otras regiones. Como ya ha sido mencionado con anterioridad, el motivo tras este fenómeno parece ser la falta de apoyo social que han recibido los certificados en sus lugares de emisión, tanto por el público general como por la comunidad LGBT.

De acuerdo con Lunsing y Tamagawa, la pervivencia de un sistema heteronormativo en Japón supone una barrera demasiado grande para que un cambio legislativo a nivel nacional sea viable en primera instancia. El establecimiento de un certificado de pareja en todas las regiones del país no afectaría de forma directa a la noción tradicional de familia; no obstante, transgrediría el sistema hetero-patriarcal y generaría por tanto conflicto. Para que el certificado fuese aceptado como un desarrollo

56 Shirakawa, T. (2016).

57 Por ejemplo, cuando la ordenanza del distrito de Shibuya estaba a punto de ser aprobada, Shūji Minato - un miembro de la sección de Tokio del LDP—afirmó que en caso de que esto sucediera "[...] nuestro próximo alcalde la revocaría" [Yasuo, T. (2017): 22].

58 Yasuo, T. (2017): 13.

59 La ausencia de grupos políticos activistas LGBT a nivel nacional también ha posibilitado la pasividad por parte del LDP en lo que concierne a los derechos de las minorías sexuales. Si bien es cierto que las interacciones entre los gobiernos locales y los miembros del colectivo han sido relevantes para el establecimiento de los certificados de pareja del mismo sexo, lo cierto es que no hay asociaciones políticas dirigidas por minorías sexuales que tengan un lobby sobre el discurso político que les concierne [Yasuo, T. (2017): 16-26]. 
positivo por el público general, dicho sistema debería desmantelarse de forma previa - una premisa difícilmente realizable en el futuro inmediato tanto de Japón como de la mayoría de los países ${ }^{60}$.

Por otro lado, algunos grupos dentro de la comunidad LGBT han rechazado la aprobación de estos certificados de pareja del mismo sexo puesto que consideran que se trata de una "media medida" 61 . Si bien para algunos activistas y miembros de las minorías sexuales los certificados están promoviendo la igualdad de género y el respeto a la diversidad, lo cierto es que muchos otros consideran que generan un contexto de desigualdad que plantea numerosos problemas ${ }^{62}$.

Los primeros defensores de los certificados habían concebido su creación para todo tipo de parejas a fin de acabar con el heteronormativismo. Sin embargo, el sistema se ha establecido solo para las uniones entre personas del mismo sexo. Al ser un recurso exclusivo de las parejas homosexuales, algunos autores consideran que estos certificados podrían estar estigmatizándolas al situar a sus integrantes en una condición de "ciudadanos de segunda". Visibilizar a las minorías sexuales a través de sistemas que refuerzan su otredad podría aumentar la persecución social de estas personas. Incluso, podría dificultar aún más su coming out y el desarrollo del activismo LGBT en el país ${ }^{63}$.

Debe considerarse además que los certificados carecen de valor legal real al no ser documentos vinculantes. Puesto que no quedan recogidos en la legislación nacional, depende exclusivamente de las diversas empresas y entidades el aceptarlos o no como prueba oficial de la unión de sus integrantes ${ }^{64}$. Solo las instituciones que dependen directamente de las administraciones regionales donde han sido aprobados tienen por obligación reconocerlos como tal. Por ejemplo, en la ciudad de Iga, las parejas poseedoras del certificado son aceptadas como miembros familiares en caso de hospitalización solo en el Hospital General de la ciudad y no en otros centros ${ }^{65}$.

Así mismo, debe tenerse en consideración que el certificado de pareja del mismo sexo implica hacer coming out, tanto ante la administración encargada de su emisión como para las instituciones en las que se trate de utilizar. En una sociedad en la que no se ha terminado de desarrollar un contexto de comprensión y aceptación de las minorías sexuales, el certificado puede suponer por ello un riesgo antes que una ventaja al no ofrecer una protección legal real a quienes lo obtienen. Algunos estudios muestran que el temor a hacer coming out no es el principal motivo por el cual no se ha propagado el uso del certificado en las zonas donde ha sido establecido. No obstante, no deja de ser un factor relevante si se considera la "vida secreta" que llevan en el país la mayoría de los miembros de la comunidad $\mathrm{LGBT}^{66}$.

Aunque algunas fuentes parecen indicar que las minorías sexuales valoran positivamente la representación legal que otorgan los certificados ${ }^{67}$, lo cierto es que a ni-

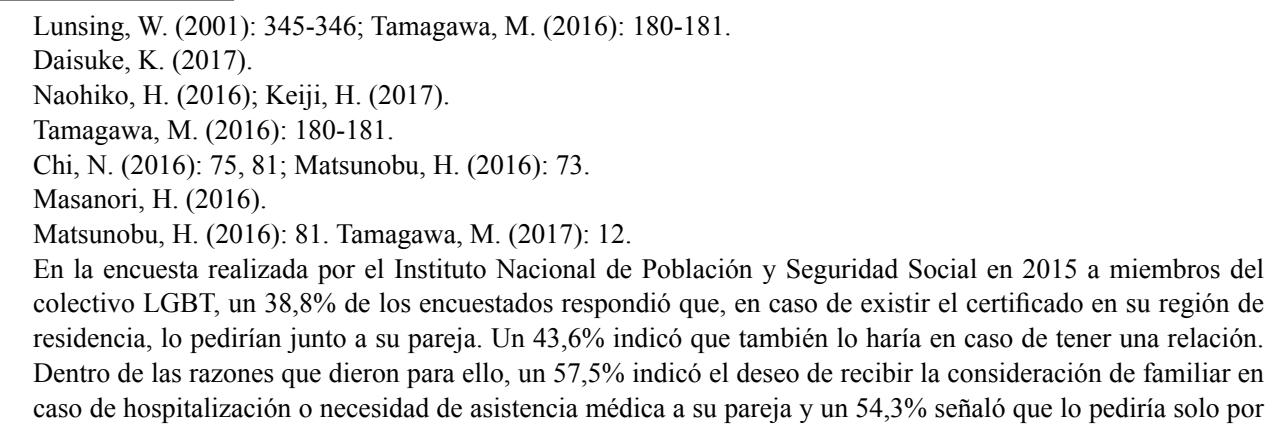
colectivo LGBT, un 38,8\% de los encuestados respondió que, en caso de existir el certificado en su región de residencia, lo pedirían junto a su pareja. Un 43,6\% indicó que también lo haría en caso de tener una relación. Dentro de las razones que dieron para ello, un 57,5\% indicó el deseo de recibir la consideración de familiar en caso de hospitalización o necesidad de asistencia médica a su pareja y un $54,3 \%$ señaló que lo pediría solo por 
vel práctico esta consideración es diferente. En un estudio realizado por Matsunobu durante el Tokyo Rainbow Parade de 2015, los encuestados indicaron que el mayor motivo para no solicitar o utilizar el certificado era la ausencia de problemas hasta el momento (45\%) o la falta de oportunidades para usarlo (28\%). Como se ha analizado anteriormente, la ausencia de trabas legales a las parejas homosexuales no es real. Por ello, este tipo de respuestas parecen indicar que el colectivo LGBT no valora que los certificados tengan una dimensión práctica efectiva. A diferencia de otros países donde se dio una gran emisión de certificados tras su aprobación, resulta llamativo que apenas se hayan dado casos en las regiones donde se ha establecido en Japón. Por ejemplo, desde su aprobación en noviembre de 2015 hasta diciembre de 2017 , tan solo 25 parejas habían solicitado en Shibuya el documento ${ }^{68}$.

\section{Conclusión}

Como reflexión final, puede afirmarse que todavía existen impedimentos políticos y sociales en Japón para el establecimiento de un certificado de pareja del mismo sexo a nivel nacional. Por un lado, es necesario tener en consideración la fuerte oposición que la medida recibiría por parte del gobierno conservador del LDP. Por otro, se debe tener en cuenta la falta de apoyo social que esta acusaría ante la pervivencia de un modelo heteronormativo en la sociedad que promueve la discriminación de las minorías sexuales, ya sea en el entorno público o privado. Más aún, se debe valorar el desencanto que la medida ha generado entre las minorías sexuales en sus lugares de aprobación, tanto por sus limitaciones legales como formales.

Con todo, el establecimiento del certificado de pareja del mismo sexo en ciertas regiones de Japón ha sido posible gracias a las iniciativas tomadas por sus administraciones locales respondiendo a los intereses de ciertos particulares y empresas y no gracias a la movilización del colectivo LGBT en defensa de sus derechos. Esta ausencia de activismo por parte de las minorías sexuales será un factor determinante para la difusión de los certificados a otras regiones y el futuro desarrollo de sus derechos en otros ámbitos legales. Por tanto, aunque todavía es temprano para evaluar el impacto de los certificados en la sociedad japonesa, puede afirmarse que su efecto a nivel práctico en la vida de los miembros de la comunidad LGBT ha sido por el momento escaso.

El principal logro de los certificados emitidos por los gobiernos locales ha sido mejorar la percepción que las minorías sexuales tienen de sí, ya que al ser documentos oficiales han otorgado cierta "aprobación social" a las parejas del mismo sexo. Al promover que los miembros de la comunidad LGBT participen activamente de la vida social y busquen su visibilización, podrían estar contribuyendo a acabar con la discriminación otonashi. Por ello, es probable que la difusión progresiva de los certificados a diferentes regiones de Japón genere poco a poco el cambio social necesario para su establecimiento a nivel nacional.

No obstante, este proceso se producirá de forma lenta considerando la gran dificultad que supone tanto el visibilizar la discriminación que las minorías sexuales padecen como hacer frente a la oposición del gobierno central y una parte de la

tratarse de un avance en su reconocimiento legal como familia. [NHK (2015)].

68 Matsunobu, H. (2016): 81; Waaldijk, K. (2001): 449; Asahi (2017). 
población fuertemente influenciada por el sistema hetero-patriarcal. La implementación a nivel estatal de los certificados de pareja del mismo sexo es una medida que, de tomar forma, lo hará en un horizonte todavía lejano.

\section{Bibliografía}

ACNUR (2014): La Protección Internacional de las Personas LGBTI. México: ACNUR, pp. 11-12. https://www.acnur.org/fileadmin/Documentos/Publicaciones/2014/9872.pdf?view $=$ [Consulta: 11/02/2019].

Arai, Yuki (2014): "Is Japan Ready to Legalize Same-Sex Marriage". En Asian-Pacific Law \& Political Journal, 16, 1, pp.122-155. http://heinonline.org/HOL/Page?handle=hein.journals/aplpj16\&div $=8 \& g$ _sent $=1 \&$ casa_token $=\&$ collection=journals [Consulta: $17 / 01 / 2018]$.

Asahi (2017): "Dōsei pātonā shōmei dai 1-gō no futari ga kaishō shibuyaku ni todokede". En Asahi Shinbun, 27/12/2017. https://www.asahi.com/articles/ASKDW51KFKDWUTIL03D.html [Consulta: 28/01/2018].

Ayuntamiento del distrito de Setagaya (2015): Setagaya-ku pātonāshippu no sensei no toriatsukai ni kansuru yōkō [página WEB]. http://www.city.setagaya.lg.jp/kurashi/101/167/1871/d00142701.html [Consulta:13/02/2018].

Ayuntamiento del distrito de Shibuya (2015): Shibuya-ku danjo byōdō oyobi tayō-sei wo sonchō suru shakai wo suishin suru jōrei [página WEB]. https://www.city.shibuya.tokyo. jp/est/oowada/partnership.html [Consulta:13/02/2018].

Barberán, Francisco /Domingo, Rafael (2006): El Código Civil Japonés. Madrid: Thomson Aranzadi.

Chi, Naomi (2016): "What is at the End of the Rainbow?: Prospects and Challenges for Sexual Minorities in Japan”. En Nenpō kōkyō seisakugaku, 10, Hokkaido, pp.75-85.

Daisuke, Kikuchi (2017): "Tokyo Rainbow Pride participants march for 'change' in LGBT recognition". En The Japan Times, 08/05/2017. http://www.japantimes.co.jp/ news/2017/05/08/national/social-issues/tokyo-rainbow-pride-participants-march-change-lgbt-recognition/\#.WRGoP1OLSu4 [Consulta:28/01/2018].

Dentsu (2015): Dentsū daibāshiti rabo ga "LGBT chōsa 2015” wo jisshi - LGBT ichiba kibo o yaku 5. 9 chō-en to sanshutsu [página WEB]. http://www.dentsu.co.jp/news/release/2015/0423-004032.html [Consulta:20/01/2018].

e-Gov: Kōei jūtaku-hō [base de datos en línea], 2016. http://elaws.e-gov.go.jp/search/elawsSearch/elaws_search/lsg0500/detail?lawId=326AC1000000193 [Consulta 12/03/2019].

EUR-Lex: "Carta de los Derechos Fundamentales de la Unión Europea". En Diario Oficial de la Unión Europea, C 202/389 [base de datos en línea], 2016. https://eur-lex.europa.eu/ legal-content/ES/TXT/?uri=CELEX\%3A12016P\%2FTXT [Consulta: 11/02/2019].

Hawkins, Joseph Raymond (1999): “A Historical and Theoretical Overview of Same-Sexuality and Aesthetics in Japan". En Invisible People: An Ethnography of Same-Sexuality in Contemporary Japan. Tesis Doctoral, Universidad de California del Sur, vol. I, pp.94162.

Hitofude, Hanpo (2017): "Pātonāshippu = Kusakabe Motomi/ Hokkaidō". En The Mainichi Shimbun, 17/04/2017. https://mainichi.jp/articles/20170417/ddl/k01/070/037000c [Consulta:28/01/2018].

International Commission of Jurists: Principios de Yogyakarta sobre la Aplicación de la Le- 
gislación Internacional de Derechos Humanos en Relación con la Orientación Sexual y la Identidad de Género [base de datos en línea], 2007. http://www.unhcr.org/refworld/ docid/48244e602.html [Consulta: 11/02/2019].

Itakura, Kyohei (2015): "Making Japan 'Out-and-Proud' Through Not-Yet-Consensual Translation: A Case Study of Tokyo Rainbow Pride's Website”. En Queer Cats Journal of LGBTQ Studies, 1, 1, pp.3-30.

Itō, Hiroki (2018): "Seiteki shōsū-sha kappuru hirogaru nintei seido-Fukuoka-shi dōnyūōreja= Motomi Kusakabe/ Hokkaidoo, de forma previa r que pus CIt.o a consecuencia de la influencia ejercida por las otras region e zenkoku 7 reime", The Asahi Shimbun, Tokyo (Edición impresa de la tarde), 14/02/2018, p.11.

Itō, Mayu (2017): "Shiki ageta dōsei kappuru ni seido no kabe nyūin ya tenkyo de hādoru". En The Mainichi Shimbun, 29/03/2017. http://www.asahi.com/articles/ASK3T7L3GK3TTIPE01D.html [Consulta: 19/12/2017].

Kazuhiro, Sekine (2016): "Sapporo LGBT activists to take fight for equality to city officials". En The Asahi Shimbun, 8/4/2016. http://www.asahi.com/ajw/articles/AJ201604080063. html [Consulta: 23/10/2016].

Keiji, Hirano (2017): "LGBT advocates push for nationwide recognition of same-sex marriage". En The Japan Times, 07/12/2017. https://www.japantimes.co.jp/news/2017/12/07/ national/social-issues/lgbt-advocates-push-nationwide-recognition-sex-marriage/\#.Wik QLQxF-V [Consulta:26/01/2018].

LDP (2018): A History of the Liberal Democratic Party [página WEB], 2018. https://www. jimin.jp/english/about-ldp/history/index.html [Consulta: 12/2/2018].

Lunsing, Wim (2001): Beyond common sense: Sexuality and gender in contemporary Japan. Londres, Nueva York, Baréin: Kegan Paul, p.210.

Lunsing, Wim (2005): "LGBT rights in Japan”. En Peace Review: A Journal of Social Justice, 17, 2-3, pp. 143-148. < doi.org/10.1080/14631370500332858> [Consulta: 12/03/2019].

Mariko, Tai (2015): "License to Love". En Nikkei Asian Review, 19/11/2015. https://asia. nikkei.com/magazine/20151119-SOMETHING-TO-PROVE/Life-Arts/License-to-love [Consulta: 10/12/2017].

Masanori, Hiuchi (2016): "City in Mie Prefecture to Recognize Same - Sex Partnerships in April". En The Asahi Shimbun, 01/03/2016. https://www.japanbullet.com/ news/city-in-mie-prefecture-to-recognize-same-sex-partnerships-in-april [Consulta: 10/12/2017].

Masaru, Sato (2016): “Commentary/ Masaru Sato: Foreign Ministry could learn from Panasonic about LGBT issues”. En The Asahi Shimbun 25/04/2016. http:/www.asahi.com/ ajw/articles/AJ201604250004.html [Consulta:18/05/2017].

Matsunobu, Hiromi (2016): “Kekkon no 'shakai-teki shōnin' to shite no dōsei pātonāshippu”. En Komazawa shakai-gaku kenkyū, 48, Tokyo, pp. 71-87.

McLelland, Mark (2005): "Salarymen doing queer. Gay men and the heterosexual public sphere”. En McLelland, Mark/ Dasgupta, Romit (eds.), Genders, transgenders and sexualities in Japan. Londres, Nueva York: Routledge, pp.96-110.

McLelland, Mark/ Suganuma, Katsuhiko (2009): "Sexual minorities and human rights in Japan: an historical perspective". En The International Journal of Human Rights, 13, 2-3, pp.329-343. < doi.org/10.1080/13642980902758176> [Consulta: 15/01/ 2018].

Miki, Keiko (2017): LGBT wo shirou. Osaka: Atelier M.

Nagayasu, Shibun (2015): "Dai 19 wa seimeihoken-Dōsei kappuru haijo no shōchō ga ugoita". En Yomiuri online, 11/05/2015. https://yomidr.yomiuri.co.jp/article/20151105-OYTEW55300/ [Consulta: 10/12/2017]. 
Naohiko, Hatta (2016): “Transgender man helps Shibuya make a difference". En The Japan Times, 13/07/2016. http://www.japantimes.co.jp/news/2016/07/13/national/social-issues/transgender-man-helps-shibuya-make-difference/\#.WRGoUFOLSu4 [Consulta:28/01/2018].

NHK (2015): LGBT tōjisha 2600 nin no koe kara [página WEB]. http://www.nhk.or.jp/d-navi/link/lgbt/\#hajime [Consulta:20/01/2018].

Nikkei (2016): "Select industries begin courting LGBT couples". En Nikkei Asian review, 20/02/2016. https://asia.nikkei.com/Business/Trends/Select-industries-begin-courtingLGBT-couples?page=1 [Consulta: 27/01/2018].

Shirakawa, Tōko (2016): "Nihon wa kawatta? Kawaranai? LGBT no ofutari niwa o kikimashita". En Yomiuri online, 02/05/2016. http://www.yomiuri.co.jp/komachi/plus/spice/20160428-OYT8T50039.html [Consulta:10/12/2017].

Shusuke, Murai (2015): “Tokyo's Shibuya and Setagaya wards issue first same-sex partnership papers". En The Japan Times, 05/11/2015. http://www.japantimes.co.jp/ news/2015/11/05/national/social-issues/shibuya-set-issue-first-certificates-recognizing-sex-couples/\#.WQhJVVOLSu4 [Consulta: 27/01/2018].

Sørensen, Johanne D. (2016): The Deinstitutionalization of Japanese Marriage?. Tesis de Máster dirigida por Ingemar Ottosson, Centre for East and South-East Asian Studies, Universidad de Lund, pp. 1-57.

Suganuma, Katsuhiko (2006): "Festival of Sexual Minorities in Japan: A Revival of the Tokyo Lesbian \& Gay Parade in 2005". En Intersections: Gender, History and Culture in the Asian Context, 12. http://intersections.anu.edu.au/issue12/katsuhiko.html [Consulta: 18/01/2018].

Tamagawa, Masami (2016): “Same-sex marriage in Japan”. En Journal of GLBT Family Studies, 12, 2, pp. 160-187. < doi.org/10.1080/1550428X.2015.1016252>.

Tamagawa, Masami (2017): "Coming out of the closet in Japan: An exploratory sociological study". En Journal of GLBT Family Studies, pp. 1-31. < doi.org/10.1080/155042 8X.2017.1338172> [Consulta 02/03/2018].

Tsuji, Kenji (2016): “Gay man from Gap hired to head Shibuya's LGBT programs". En The Asahi Shimbun, 03/10/2016. http://www.asahi.com/ajw/articles/AJ201610030043.html [Consulta:12/02/2018].

Waaldijk, Kees (2001): "Small change: How the road to same-sex marriage got paved in the Netherlands". En Wintemute, Robert/ Andenæs, Mads Tønnesson (eds.), Legal Recognition of Same-Sex Partnerships. Portland, Oxford: Hart Publishing, pp.437-464.

Yasuo, Takao (2017): “The Politics of LGBT Policy Adoption: Shibuya Ward's Same-Sex Partnership Certificates in the Japanese Context". En Pacific Affairs, 90, 1, pp.7-27. <doi: 10.5509/20179017> [Consulta: 12/03/2019]. 